\title{
Anatomy of coronary sinus ostium
}

\author{
J. Zhivadinovik, M. Papazova, N. Matveeva, A. Dodevski, B. Zafirova \\ Institute of Anatomy, Medical Faculty, Skopje, R. Macedonia
}

[Received: 22 July 2015; Accepted: 13 August 2015]

Background: The coronary sinus is the main cardiac vein and it has become a clinically important structure especially through its role in providing access for different cardiac procedures.

Materials and methods: The study was carried out on 100 randomly selected adult human cadaver hearts fixed in 10\% formalin. The transverse and craniocaudal diameters of the coronary sinus ostium (CSO) were directly measured. The presence of the Thebesian valve was noted and the anatomical details of the valve were documented in each case in terms of the shape and extent of coverage of the CSO. Results: Considerable variations in the diameter of the CSO were observed. The mean craniocaudal diameter of the CSO was $8.1 \pm 1.51 \mathrm{~mm}$, and the mean transverse diameter was $7.67 \pm 1.72 \mathrm{~mm}$. Heart specimens without Thebesian valve tended to have larger ostia. The mean craniocaudal diameter and the mean transverse diameter of the CSO were statistically larger in the specimens without Thebesian valves ( $p=0.000$ and $p=0.001$, respectively).

Conclusions: The Thebesian valves were observed in 86 hearts, and a wide variety of their morphology was seen. The majority of the Thebesian valves were semilunar in shape (74.42\%). The extent to which the valve covered the ostium was variable, including remnant valves that covered $<15 \%$ of the CSO (35\%), and valves that were large and covered at least $75 \%$ of the CSO (22.09\%). In 3 specimens the valve completely occluded the ostium. (Folia Morphol 2016; 75, 2: 264-267)

Key words: Thebesian valve, coronary sinus ostium, variations

\section{INTRODUCTION}

The coronary sinus (CS) is the main cardiac vein and it has become a clinically important structure especially through its role in providing access for different cardiac procedures.

The $\mathrm{CS}$ is a wide venous channel, about $2-3 \mathrm{~cm}$ long, situated in the left, diaphragmatic part of the coronary sulcus, covered by muscular fibres from the left atrium. It ends in the right atrium, between the opening of the inferior vena cava and the atrioventricular aperture. Its orifice (coronary sinus ostium [CSO]) is $5-15 \mathrm{~mm}$ in diameter and it is often guarded by a fold of endocardial tissue, an embryological remnant of the caudal portion of the right valve of the embryonic sinoatrial orifice, termed the valve of the CS or Thebesian valve (TV) $[9,10]$.

Catheterisation of CS is often used in the mapping and ablation of atrioventicular accessory pathways, the CS flow studies, the retrograde perfusion of cardioplegic solutions and cardiac resynchronisation therapy [9]. Cannulation of the CS during these procedures may be complicated by the obstruction due to the morphology of TV $[3,6,4,7,11]$. Therefore, studies on morphology of the $\mathrm{CSO}$ and its valve have potential practical implications. 
Table 1. Diameters of coronary sinus ostium (CSO)

\begin{tabular}{lcccc}
\hline Diameters of ostium & $\begin{array}{c}\text { CSO } \text { with absent valve } \\
(\mathbf{n}=\mathbf{1 4})\end{array}$ & $\begin{array}{c}\mathbf{C S O} \text { with valve } \\
(\mathbf{n}=\mathbf{8 6})\end{array}$ & $\begin{array}{c}\text { Test of significance } \\
\text { for differences of means }\end{array}$ & $\begin{array}{c}\text { Total } \\
(\mathbf{n}=100)\end{array}$ \\
\hline Transverse diameter & $9.28 \pm 2.43$ & $7.41 \pm 1.43$ & $\mathrm{P}=0.000$ & $7.67 \pm 1.72$ \\
& $(5-15)$ & $(5-12)$ & & $(5-15)$ \\
Craniocaudal diameter & $9.36 \pm 1.9$ & $7.89 \pm 1.34$ & $\mathrm{P}=0.001$ & $7.67 \pm 1.72$ \\
& $(5-12)$ & $(5-12)$ & & $(5-15)$ \\
\hline
\end{tabular}

The aim of this study was to present the anatomical features of the CSO and the anatomical variations of TV with regards to its shape and extent of coverage of the CSO in human cadaveric hearts.

\section{MATERIALS AND METHODS}

The study was carried out on 100 adult human cadaver hearts fixed in $10 \%$ formalin, randomly selected from the collection of the Institute of Anatomy at the Medical Faculty in Skopje, R. Macedonia. The specimens were removed together with the proximal portions of the great vessels: aorta, pulmonary trunk and superior vena cava and opened using a standard anatomical dissection, taking care that the TV and CSO were kept intact. The right atrial wall was opened from the posterior plane, by dissecting through the line from the orifice of superior vena cava to the orifice of inferior vena cava. The dissection continued to the apex of the heart. The left side of the heart was dissected along the margo obtusus.

The hearts were weighed with digital scale. The transverse and craniocaudal diameters of the CSO were directly measured to the nearest millimetre using a calliper. The measurements were undertaken with the heart held in an anatomical position; superior vena cava and inferior vena cava being in axis parallel to the longitudinal axis of the body and the longitudinal axis of the heart, maintained in a semihorizontal position. The measurements were undertaken by two observers to reduce the chance of error. The presence of the TV was noted and the anatomical details of the valve were documented in each case in terms of the shape and extent of coverage of the CSO. According to their shapes, the valves have been classified as semilunar, fenestrated and band-like. According to the extent of coverage of the CSO the valves were classified as covering less than $15 \%$ of the CSO, between $15 \%$ and $75 \%$ and more than $75 \%$ of the ostium.

\section{Statistical analysis}

Obtained data were statistically analysed with descriptive analyses and presented as mean \pm standard deviation and range. Student's t-test was used for statistical comparison between ostia with valve and ostia without valve. The significance level was set at $5 \%$. Statistical analysis was performed using the SPSS (version 20, Chicago, IL, USA).

\section{RESULTS}

Demographic data were available from all 100 examined specimens. The mean age was $57.6 \pm 14.8$ years (range 20-87). Seventy two of the specimens were from male cadavers and 28 were from female cadavers. The average weight of the hearts was $358.40 \pm 89.30 \mathrm{~g}$ (range 220-950).

Considerable variations in the diameter of the CSO were observed. The mean craniocaudal diameter of the CSO was $8.1 \pm 1.51 \mathrm{~mm}$, and the mean transverse diameter was $7.67 \pm 1.72 \mathrm{~mm}$. Heart specimens without TV tended to have larger ostia. The mean craniocaudal diameter and the mean transverse diameter of the CSO were statistically larger in the specimens without TV ( $p=0.000$ and $p=0.001$, respectively) (Table 1).

The TV were observed in 86 hearts, and a wide variety of their morphology was seen (Figs. 1-4). The majority of the TV were semilunar in shape (74.42\%). The extent to which the valve covered the ostium was variable, including remnant valves that covered $<15 \%$ of the CSO (35\%), and valves that were large and covered at least $75 \%$ of the CSO $(22.09 \%)$. In 3 specimens the valve completely occluded the ostium (Table 2 ).

\section{DISCUSSION}

Anatomical variations of the CSO and the TV are numerous, and, as many authors have reported, the valve at the entrance to the CS could pose difficulties in cannulation of the CS [3, 4, 6, 7, 9-12]. It has been suggested that the role of the TV in normal physiology is to prevent the regurgitation of blood into the 


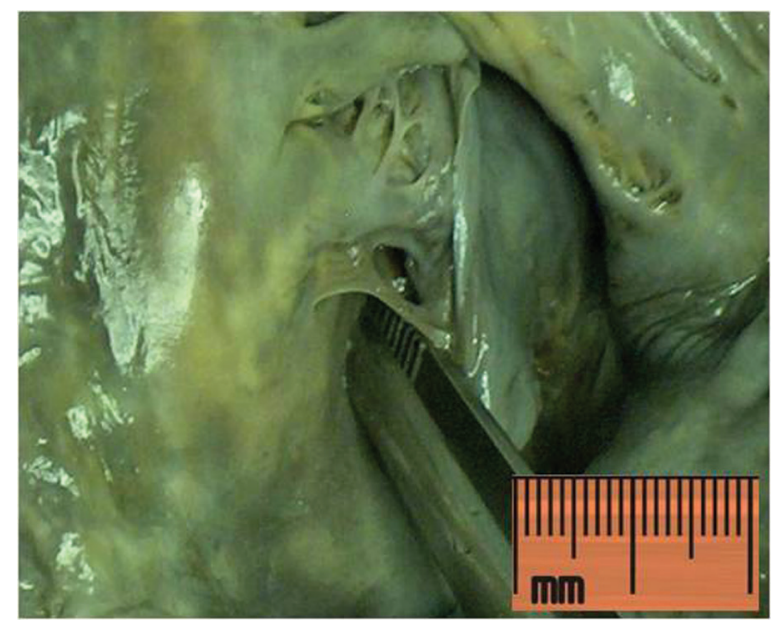

Figure 1. Fenestrated Thebesian valve.

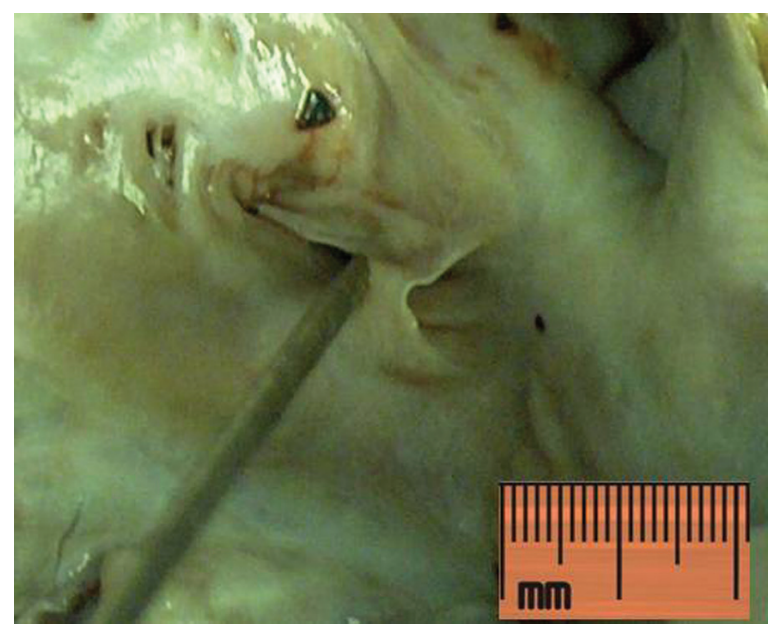

Figure 2. Band-like Thebesian valve.

CS during atrial systole. Relatively small number of studies describes echocardiography imaging of the normal TV. Presumably due to its thin nature and small size it is usually visualised occasionally $[1,2,8]$.

The TV is always described in anatomy textbooks, but it is hard to find detailed systematic studies of its structure and function. There are different classifications that describe the morphology of the valve [3]. According to Karaca et al. [6] the TV can be present or absent. The first one may cover different degree of the ostium (0-100\%), and depending on the shape it can be crescent (31\%), semilunar $(29 \%)$ or bandshaped (4\%). They found fenestrated valves in $4 \%$ of their cases. In 1991, Jatene et al. [5] published an anatomic study of the TV in 94 human hearts [2].

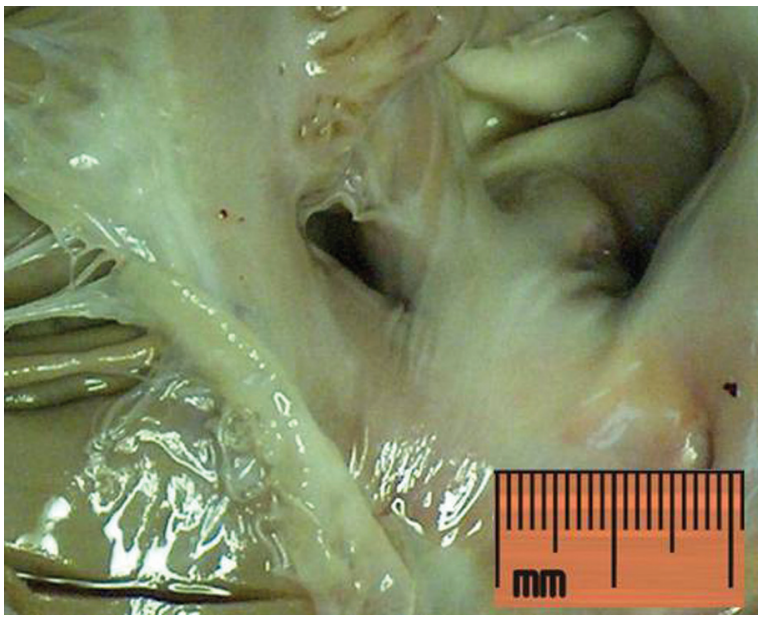

Figure 3. Thebesian valve covering less than $15 \%$ of the coronary sinus ostium.

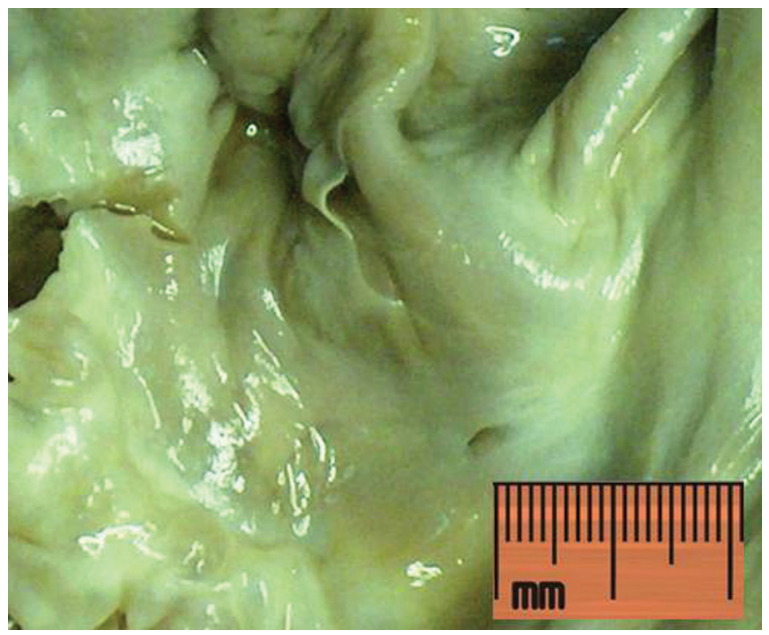

Figure 4. Thebesian valve covering more than $75 \%$ of the coronary sinus ostium.

Table 2. Morphological features of Thebesian valve

\begin{tabular}{llcc}
\hline Thebesian valve & & $\mathbf{N}$ & $\%$ \\
\hline Shape: & Semilunar & 64 & 74.42 \\
& Band-like & 15 & 17.44 \\
& Fenestrated & 7 & 8.13 \\
& Total & 86 & 100 \\
& $<15 \%$ & 30 & 35 \\
Extent of coverage & $15-75 \%$ & 37 & 41.86 \\
of the coronary & $>75 \%$ & 19 & 22.09 \\
sinus ostium: & Total & 86 & 100 \\
& & & \\
\hline
\end{tabular}

They described four categories of development of the valve: absent, residual - covering less than 15\% 
of the CSO, partial - covering more than $15 \%$ of the CSO and trabecular or double. Mak et al. [10] published the results from 75 autopsied human hearts (54 with organic heart disease), that confirmed a wide variety of TV patterns. The valve was present in $73 \%$ of examined hearts, and the most common shape was semicircular (65.4\%). Fenestrations were observed in $26 \%$ of valves, $16 \%$ of hearts showed a valve that covered more than $75 \%$ of the ostium. The transverse and craniocaudal dimensions of the CSO in hearts with TV were significantly smaller compared to those specimens with no TV. Pejkovic et al. [11] analysed 150 human heart specimens and they found TV in $80 \%$ of cases. It covered one-third of the ostium in $29 \%$ of cases, one-half in another $27 \%$, and two-thirds in $14 \%$. The whole ostium was covered in $5 \%$ of cases. Ghosh et al. [3] reported an anatomical study on 150 human hearts where TV were present in $79 \%$ of the specimens. They classified the TV according to their shape as semilunar, fenestrated and biconcave band-like. Semilunar was the most common type. They defined the non-fenestrated (semilunar, biconcave band-like) and non-membranous (fibromuscular, fibrous, muscular) valves covering $>75 \%$ of the CS ostium as an obstructive TV that could interfere with the cannulation of the CS. The presented data are in concordance with our results. All these studies confirm the significant variability in the presence and arrangement of the $\operatorname{TV}[3,4,6,7,9-12]$. This study is the first one of this kind carried out in our country on our population. We have simplified the previously published classifications, and have classified the valves according to their shapes, in semilunar, fenestrated and band-like. Depending on the extent of coverage of the CSO the valves were classified as covering less than $15 \%$ of the CSO, between $15 \%$ and $75 \%$ and more than $75 \%$ of the ostium. Our results showed that the TV was present in $86 \%$ of hearts. Specimens without TV tended to have larger ostia. The mean craniocaudal diameter and the mean transverse diameter of the CSO were statistically larger in the specimens without TV $(p=0.000$ and $p=0.001$, respectively). Majority of the TV were semilunar in shape $(74.42 \%)$. The extent to which the valve covered the ostium was variable, but the majority of the valves covered between $15 \%$ and $75 \%$ of the CSO ( $41.86 \%)$. In 3 specimens the valve completely occluded the ostium. The valves that are not fenestrated and occlude more than $75 \%$ of the CSO can be considered as obstructive and could lead to unsuccessful CS cannulation.

\section{CONCLUSIONS}

This study was made on formalin fixed hearts; hence the changes in cardiac dimensions may have occurred. Despite these limitations, we can conclude that the TV was present in $86 \%$ of the specimens; the most common shape was semilunar (84.8\%), and the dimensions of the CSO were significantly larger in the hearts where the valve was absent. Large TV that cover more than $75 \%$ of CSO and could potentially complicate the cannulation of the CS were seen in $19 \%$ cases; three of them completely covered the OSC. The knowledge of these anatomical variations of the CSO and the TV may be useful in overcoming the difficulties in clinical practice.

\section{REFERENCES}

1. Anh DJ, Eversull CS, Chen HA, Mofrad P, Mourlas NJ, Mead RH (2008) Characterization of human coronary sinus valves by direct vizuelization during biventricular pacemaker implantation. Pacing Clin Electrophysiol, 31: 78-82.

2. D'Cruz IA, Shirwany A (2003) Update on echocardiography of coronary sinus anatomy and physiology. Echocardiography, 20: 87-95.

3. Ghosh KS, Raheja S, Tuli A (2014) Obstructive Thebesian valve: anatomical study and implications for invasive cardiologic procedures. Anat Sci Int, 89: 85-94.

4. Habib A, Lachman N, Christensen K, Asirvatham S (2009) The anatomy of the coronary sinus venous system for the cardiac electrophysiologist. Europace, 11: 15-21.

5. Jatene M, Jatene F, Costa R (1991) Anatomical study of the coronary sinus valve - Thebesius valve. Chest, 100 (suppl.): 90S.

6. Karaca M, Bilge O, Dinckal MH, Ucerler H (2005) The anatomic barriers in the coronary sinus: implications for clinical procedures. J Interv Card Electrophysiol, 14: 89-94.

7. Kautzner J (2009) Thebesian valve: the guard dog of the coronary sinus? Europace 11: 1136-1137.

8. Kebble W, Mohiaddin R (2008) Technical failure to perform cardiac resynchronization therapy: Use of cardiac magnetic resonance imaging techniques to clarify a left-sided superior vena cava and coronary sinus morphology. Can J Cardiol, 24: 589-590.

9. Loukas M, Clarke P, Tubbs RS, Kolbinger W (2008) Adam Christian Thebessius, a historical perspective. Int J Cardiol, 129: 138-140.

10. Mak GS, Hill AJ, Moisiuc F, Krishnan SC (2009) Variations in Thebesian valve anatomy and coronary sinus ostium: implications for invasive electrophysiology procedures. Europace, 11: 1188-1192.

11. Pejkovic B, Krajnc I, Anderhuber F, Kosutic D (2008) Anatomical variations of the coronary sinus ostium area of the human heart. J Int Med Res, 36: 314-321.

12. Singh JP, Houser S, Heist EK, Ruskin JN (2005) The coronary venous anatomy: a segmental approach to aid cardiac resynchronization therapy. J Am Coll Cardiol, 46: 68-74. 